\title{
AN INTEGRATED COMPUTER AIDED ENGINEERING TOOL FOR AIRCRAFT TRANSPARENCY DESIGN ANALYTICAL DESIGN PACKAGE - ADP
}

\author{
J.E. Wuerer \\ The MacNeal-Schwendler Corporation \\ Costa Mesa, California \\ M. Gran \\ Air Force, Wright Laboratory \\ Wright-Patterson AFB, Ohio \\ T.W. Held \\ University of Dayton Research Institute \\ Dayton, Ohio
}

\begin{abstract}
The Analytical Design Package (ADP) has been developed as a part of the Air Force Frameless Transparency Program. ADP is an integrated design tool consisting of existing analysis codes and Computer Aided Engineering (CAE) software. The objective of the ADP is to develop and confirm an integrated design methodology for frameless transparencies, related aircraft interfaces, and their corresponding tooling. The application of this methodology is intended to generate a high confidence for achieving a qualified part prior to mold fabrication.

ADP is a customized integration of analysis codes, CAE software and material information databases. The primary CAE integration tool for the ADP is MSC/PATRAN, a commercial-offthe-shelf (COTS) software tool. The open architecture of MSC/PATRAN allows customized installations with different application modules for specific site requirements. Integration of material databases allows the engineer to select a material and those material properties are automatically input into the relevant analysis code. The ADP materials database supports two independent schemas: (1) CAE Design Properties and (2) Processing and Test Data.

The design of the ADP places major emphasis on the seamless integration of CAE and analysis modules with a single intuitive graphical interface. This tool has been designed to serve and be used by an entire project team, i.e., analysts, designers, materials experts and managers. The final version of the software was delivered to the Air Force in June 1995. The Analytical Design Package (ADP) is intended to facilitate technology transfer to industry. The analysis system is capable of a wide range of design and manufacturing applications.
\end{abstract}




\subsection{INTRODUCTION}

The Analytical Design Package (ADP) is an integrated design tool consisting of existing analysis codes and Computer Aided Engineering (CAE) software. The objective of the ADP effort is to develop and confirm an integrated design methodology for frameless aircraft transparencies. ADP analysis capabilities include: aerodynamic heating, transient thermal response, static and dynamic structure response, optical ray trace, injection molding process simulation, and an aircraft transparency related material properties modeling and databank system. The design process is iterative and capable of producing frameless transparency designs, information needed for the design of integral aircraft interfaces, and information needed to support the design of injection molding tooling and the specification of molding process parameters for specific materials.

The current version of the ADP (Version 2.1) is a second generation analysis system. The initial ADP development was initiated in 1989, References 1, 2 and 3. Since the inception of the original ADP, significant developments in both CAE and design support software relevant to aircraft transparency design have evolved. In addition, certain current design requirements, e.g., optics analysis and material properties modeling, were not addressed in the original ADP. Finally, significant advances in computing hardware have occurred making it possible to perform the required computations on workstation systems as opposed to mainframe platforms.

Recent developments in CAE design tools have introduced the ability to integrate special purpose and commercial-off-the-shelf (COTS) software in a user friendly (intuitive/interactive) environment. That is, to have a single user interface serve the primary analysis functions, specifically:

1. Modeling

- Geometric Modeling (construction and modification)

- CAD and IGES File Import

- Graphics Manipulation

- Meshing

- Loads and Boundary Conditions Specifiction

2. Materials Data Management

- Analysis Code Properties Input

- Test Data Processing and Reduction

3. Analysis Module and Module Parameter Specification

4. Results Evaluation

The design of ADP places major emphasis on the seamless integration of CAE and analysis modules with a single intuitive graphical user interface.

\subsection{ADP (Version 2.1) SCOPE}

The principal objective of the ADP development was to provide an integrated CAE tool to support the design of frameless (injection molded) aircraft transparencies. This tool has been designed to serve and be used by the entire project team, i.e., analysts, designers, materials experts, and managers. Emphasis was placed on ease of use with the need for the user to learn only one common graphical user interface.

The ADP design places emphasis on the use of standardized methods for electronic communication of data to support the CAE process. Specific examples include: (1) the ability to 
import CAD and/or IGES files to simplify geometry model development, (2) the ability to store, process and import materials property data to support the design process, and (3) to allow users (including users at different sites) to share analysis results.

Finally, the ADP design addresses the issue of cost effectiveness. Where possible, commercial off-the-shelf (COTS) software is specified. This approach alleviates the need for the owner to support and upgrade the software system as relevant new developments and improvements emerge. In addition, COTS software portability is generally maintained for common engineering work station platforms. Cost effectiveness is also addressed through ease of use and enhanced design team communication. For example, the design team has the ability to review analysis results from the perspective of their individual needs. Ease of use allows all members of the design team to communicate through direct use of the ADP to support their individual roles.

The specified analysis module requirements for ADP (Version 2.1) are listed in Table 1. ADP (Version 2.1) has been configured so as to eventually allow the evolution of an ADP based totally on commercial-off-the-shelf (COTS) software. This potential evolution is indicated as ADP/COTS in Table 1. Although ADP/COTS was not a development target for the present program, it was, however, an important planning issue for ADP supportability and had a direct impact on the ADP (Version 2.1) architecture.

The primary $\mathrm{CAE}$ integration tool for ADP (Version 2.1) and ADP/COTS is MSC/PATRAN. The open architecture of MSC/PATRAN provides the option to use several solver modules other than the baseline modules indicated in Table 1. A listing of alternative solvers which are commercially integrated with MSC/PATRAN are listed in Table 2. This flexibility of MSC/PATRAN expands the opportunity for ADP (Version 2.1) and/or ADP/COTS users to use their preferred solvers.

Releases of ADP (Version 2.1) have been developed for operation on Silicon Graphics (SGI) and Hewlett Packard (HP) UNIX based workstations. The specific operating systems used for development were SGI (IRIX 5.2) and HP (HPUX 9.03) respectively. The specific software components which define the ADP (Version 2.1) software system are listed as follows:

\section{$\underline{\text { Commercial Components }}$}

1. MSC/PATRAN (Version 1.4)

2. MSC/PATRAN Materials Selector

3. MSC/PATRAN IGES

4. MSC/PATRAN Animation

5. MSC/NASTRAN (Version 68)

6. MSC/PATRAN FEA (Version 1.4-1)

7. MSC/MVISION (Version 1.2)

8. C-MOLD (Version 4.0)

\section{Third Party Components}

1. ADP CUSTOMIZATION (Version 2.1)

2. STAHET (Version 2.0)

3. TAP (Version 2.0)

4. X3D (Version 3.71)

5. OPTRAN (Version 1.31)

A complete system requires that the user procure the commercial components relevant to the users specific needs. The third party components are delivered on a single tape and are available through the Air Force.

The overall architecture for ADP (Version 2.1) is shown in Figure 1. The primary CAE integration element of ADP (Version 2.1), MSC/PATRAN, provides the basic framework for the executive control to implement application module integration, pre-processing (modeling) capability and post-processing (results display) capability. The basic functionality of MSC/PATRAN is extended by the ADP customization, indicated by the shaded area in Figure 1. 


\subsection{ADP (Version 2.1) CUSTOMIZATION}

What does the ADP Customization consist of? This is a question that several potential ADP users have asked. Comments have been made to the effect that one is not able to determine where MSC/PATRAN ends and ADP begins. The reason for this is actually one of the advantages of using the customization features of MSC/PATRAN as the core of the ADP. Enhancements made to MSC/PATRAN via the use of PATRAN Command Language (PCL) makes all new functionality seamless. In other words, the enhancements actually appear to be a part of MSC/PATRAN.

The ADP customization may be quantified in terms of lines of code. An approximate inventory of code language and lines of code required to provide the primary functionality is listed as follows:

\section{$\underline{\text { ADP Component }}$}

Project Manager

UNIX Server

Application Interfaces
Code Language

PCL

$\mathrm{C}$ and SQL

PCL

C

FORTRAN

Total
Lines

25,000

7,000

38,000

14,000

$\underline{1,000}$

85,000

\subsection{The ADP System}

The ADP system consists of the collection of specific commercial and non-commercial software applications which are required for the design and manufacture of aircraft transparencies. These software products were then integrated with custom software to simplify the work of the engineers and analysts.

Specifically, the software products integrated for ADP have been listed in Table 1. The ADP system functionally consists of two parts: the Project Manager and the Application Interfaces. The Project Manager manages all data which the ADP user creates and accesses. It also manages the process flow between MSC/PATRAN and the various simulation tools. The Application Interfaces integrate the specific simulation tools with MSC/PATRAN.

\subsection{The Project Manager}

The Project Manager is responsible for all items that relate to project management and process flow. Under most instances, the user will no longer need to separately keep track of his files. All data within ADP are referenced with respect to a specific project or task. The user will simply select the task or project that he is interested in and begin modeling or analyzing. ADP even keeps track of where a particular user was working during his last ADP session, and places him in that same project the next time that he invokes ADP.

The Project Manager also insures that the particular analysis tool selected by the user is used exclusively on the model that was created for that specified analysis task. The Project Manager actually instructs MSC/PATRAN about which analysis tool to use with a specific model. When the user specifies to the project manager that he wants to perform an analysis, MSC/PATRAN knows exactly which analysis to perform.

Another feature of ADP is the ability for users to share data. A user can easily copy data from one project to another. The user may also copy data from the outside world (data existing outside of the ADP system) into the ADP Project Management System. 


\subsection{The Application Interface}

The Application Interface consists of all Analysis Tools as well as their forward and reverse translators to MSC/PATRAN. It also consists of the graphical forms interface used by the engineer to specify all actions performed by a specific analysis tool. Consider for example the application interface to the birdstrike analysis module, X3D. Once the Project Manager delivers the model to MSC/PATRAN, all PATRAN general functions are available to modify, refine and complete the model. When the user is ready for analysis, he selects "Analysis" on the PATRAN menu bar, and the custom X3D graphical forms are displayed. The custom forms assist the user in specifying all parameters for the type of simulation he wishes X3D to perform, and they initiate the actual job submittal and results read-in. These forms are part to the custom code developed for the ADP Applications Interface. In the case of $\mathrm{X} 3 \mathrm{D}$, the model and solution parameters are automatically provided to the forward translator, PAT3X3D. This translator converts the data into the run stream format that X3D requires. After the model and solution parameters are translated, $\mathrm{X} 3 \mathrm{D}$ is automatically submitted as a background job. Once the $\mathrm{X} 3 \mathrm{D}$ job has completed, the results are imported back into the MSC/PATRAN model by use of the reverse translator, X3DPAT3. PAT3X3D and X3DPAT3 are also parts of the custom code which make up the ADP Applications Interface.

\subsection{ADP APPLICATION MODULES}

There are a total of eight primary components of simulation functionality built into the ADP. These are listed as follows:

1. Integration, Modeling and Pre-and Post-Processing

2. Materials Data Management

3. Aerodynamic Heating Simulation

4. Thermal Response Simulation

5. Structural Performance Simulation

6. Bird Impact Response Simulation

7. Optical Performance Simulation, and

8. Injection molding Process Simulation

An overview of the functionality provided in each of these components follows.

\subsection{Integration, Modeling, and Pre-and Post-Processing}

The integration, modeling and pre- and post-processing functionality of the ADP is provided by MSC/PATRAN

MSC/PATRAN is an advanced computer aided engineering (CAE) integration tool introduced in June 1992, Reference 4. MSC/PATRAN tool has an open architecture which provides several important capabilities. These include:

1. direct software links to leading CAD systems,

2. direct software links to analysis software programs, both leading commercial and user developed and/or maintained programs,

3. pre- and post-processing capabilities to allow geometry and FEM development, as well as analysis results display,

4. an integrated subset of the materials selection capability from The MSC/MVISION family of products, and

5. a fully integrated set of MSC/PATRAN analysis modules for performing structural, thermal, computational fluid dynamic, fatigue, and other types of mechanical analyses. 
MSC/PATRAN has a user friendly "mouse activated" window environment graphical user interface (GUI) which provides execution of the analysis system without the need to remember a particular command syntax. The open system architecture and GUI provided the basic capability to build a user friendly executive control capability for the ADP.

MSC/PATRAN was specified for ADP primarily for its capability as a CAE system integration tool. However, there are several analysis modules that are fully developed and completely integrated with MSC/PATRAN. These analysis modules include:

1. MSC/PATRAN FEA for comprehensive finite element analysis,

2. MSC/PATRAN ADVANCED FEA which is a non-linear module developed jointly with HKS based on ABAQUS analysis technology,

3. MSC/PATRAN FATIGUE for durability analysis,

4. MSC/PATRAN CFD is a state-of-the-art computation fluid dynamics module for workstations,

5. MSC/PATRAN THERMAL is a state-of-the-art finite element based thermal analysis, and

6. MSC/PATRAN ANIMATION is an advanced, state-of-the-art animation capability codeveloped with Intelligent Light.

All of the above modules have potential specific relevance to the requirements of the ADP, several examples of which are shown in Table 1

\subsection{Materials Data Management}

Materials data management functionality is provided by MSC/MVISION. This materials software system provides for data storage visualization, selection and integration, Reference 5 . MSC/MVISION uses centralized relational databases organized to reflect the classes of materials information needed in the design-to-manufacturing process:

1. Material: Material source and designations, as well as extension to account for the multiple sources associated with composite materials.

2. Specimen: Detailed information about the specimens including composition and processing specifics.

3. Environment: Specific information about the experimental conditions including temperature and humidity as well as statistics and quality.

4. Properties: Mechanical, physical, thermal, and electrical properties of materials including extensions to account for anisotropy. Curves and rasterized images are represented as well.

Data stored in MSC/MVISION may be directly accessed by MSC/PATRAN via the MSC/PATRAN MATERIALS SELECTOR.

MSC/MVISION provides a very significant capability for materials database management functionality. Details of the role of MSC/MVISION in the ADP design methodology are presented in Reference 6 . The following partial list summarizes several pertinent features provided by the MSC/MVISION COTS product:

1. Complete graphical user interface (GUI) that provides visualization of all materials database functions. 
2. Customization of database schema; i.e., attribute list, relationships, etc. Easy to expand database to include more properties and more property types.

3. Units easily changed to SI or any user defined system.

4. Metadata and footnotes provided with data values that enable inclusion of material source, specimen conditions, test environment, test anomalies, etc.

5. Data can be stored and manipulated using an extensive spreadsheet functionality.

6. Database queries using engineering terminology to define search conditions.

7. Full-featured manipulation and storage of tables, graphics, and CAT scan images.

8. Strict adherence to government and industry standards.

9. Provides data importing and exporting features using the following exchange protocols:

- IGES

- PDES/STEP

- $\quad$ MSC/PATRAN Neutral File

- $\quad$ ASCII Text Spreadsheet Files

As a powerful stand-alone product, MSC/MVISION can be utilized in a variety of ways, within the design-to-manufacturing process. Generally, throughout the design process, engineers do not have a central on-line source and electronic access to high quality materials information required for analytical design assessment simulations. Often the materials information that is available is missing critical property values requiring further testing or analytical material synthesis. As a on-line source for data management, MSC/MVISION is intended to store all the available materials information pertinent to the transparency design team.

\subsection{Aerodynamic Heating Simulation}

Computations which predict the aerodynamic heating history to the transparency system for a specific aircraft configuration and mission profile are performed by the STAHET solver. The results of these computations are then used as input boundary conditions to the transient heating analysis (TAP) for the detailed transparency model, as discussed in Section 4.4.

The STAHET and TAP codes were developed to be embedded in the STAPAT (Specific Thermal Analyzer Code for Aircraft Transparencies) development. STAPAT, rather than a single computer program, is actually an analysis methodology incorporating a set of computer codes. There are 5 major elements of this methodology, specifically:

1. develop the forebody model,

2. compute the aeroheating over the transparency surface using the STAHET computer code and these models (STAHET),

3. develop the transparency finite element model (STABLD),

4. compute the transient, three dimensional transparency system temperatures using the TAP computer code and the finite element model (TAP), and

5. display the models and results using the STAPLT computer code (STAPLT).

Details of the above methodology are reported in References 7, 8, and 9. 
The above methodology posed several inconsistencies with the ADP architecture, specifically with respect to Item 1, forebody model; Item 2, transparency finite element model; and Item 5, display models and results. In ADP, all of these tasks are accomplished within MSC/PATRAN.

The general capabilities of the STAHET solver were developed in two-phases. A summary of the multiphase development is provided in Table 3. Details of the indicated capabilities, their underlying assumptions and limitations, and their implementation are presented in References 7, 8 and 9.

The integration of STAHET into the ADP product is limited to those capabilities relevant to current tactical and strategic aircraft. Capabilities relevant to hypersonic vehicle applications, i.e., Mach Number greater than about 4 and altitude greater than 100,000 feet, exist in the basic STAHET code. The implementation of this capability would require an extension of the ADP/STAHET application interface.

An important feature of the STAHET integration into ADP is the inclusion of (and ready availability of) aircraft forebody geometries. The complete file of 16 STAHET geometries has been included. Geometries relevant to conventional military aircraft include F-16, F-15, F-4, F18, and B-1B. Low RCS aircraft, missile, and several hypersonic forebodies also exist in the library. An example geometry, the F-16 forebody configuration, is shown in Figure 2.

An example of an ADP/STAHET result is provided in Figure 3. Shown is a fringe plot of aerodynamic heating rate over the portion of the forebody relevant to the transparency. The relevant aerodynamic heating parameters are then transferred to TAP where the predictions of the transient thermal response for the transparency are conducted.

\subsection{Thermal Response Simulation}

The transient thermal response analysis for the transparency structure is performed by the TAP solver. The primary end result of the analysis is the temperature field as a function of time for the detailed 3-dimensional finite element model. These data may then be queried to examine for critical temperatures and/or be directly handed off to the thermal stress finite element model via the MSC/PATRAN FEM Field Interpolator.

The general capabilities of the TAP application module are summarized in Table 4. Details of the above capabilities and their implementation are discussed in References 8 and 9.

An example geometry used to build the TAP finite element model (FEM) is shown in Figure 4. The specific geometry is for the designated "Confirmation Frameless Transparency" (CFT7A) as specified by Wright Laboratories. Example TAP results are shown in Figure 5. Shown is the temperature field, at a specific mission time point, displayed in the TAP FEM via the P3/PATRAN post-processing capability.

\subsection{Structural Performance Simulation}

Modeling support for two structural analysis solvers have been provided in ADP (Version 2.1). These are MSC/NASTRAN and MSC/PATRAN FEA.

MSC/NASTRAN is the most comprehensive FEA program in existence. It was specified on the basis that it is used, and therefore available, in many aircraft design houses. MSC/NASTRAN is extensively supported by its commercially maintained interface with MSC/PATRAN. This interface effectively supports all of the requirements for the transparency design problem. Particular strengths of MSC/NASTRAN are its ability to handle very large problems, its computational efficiency, and its many optional functionalities, Reference 10. 
MSC/PATRAN FEA is a general purpose structural analysis solver which is fully integrated with MSC/PATRAN. It supports the basic requirements for the transparency design problem. It provides a lower cost somewhat easier to use solution, Reference 11.

A comparison of the general capabilities of MSC/NASTRAN and MSC/PATRAN FEA relevant to the aircraft transparency design problem is listed as follows:

\section{Functionality}

Static Structural:

Linear Statics

Natural Frequencies, Modes

Buckling

Contact Problems, Gaps

Composites, 2-D Laminates

Coupled Thermal-Stress

Very Large Model Computational Efficiency

Coupled Aero or Hydro Elasticity

Nonlinear Materials (Rubber, Plastics)

Large Deformations

Structural Dynamics:

Linear

Large Deformation, Nonlinear Materials

Thermal:

Fixed Boundary Conditions: Transient, Steady State.

Variable B.C., Transient: Steady State

\section{$\underline{\text { MSC/NASTRAN }}$}

Yes

Yes

Yes

Yes

Yes

Yes

Yes

Yes

Yes

Yes

Yes

Yes

Yes

Yes
MSC/PATRAN FEA

Two basic structural performance simulations are needed to support the ADP design assessment for aircraft transparencies. These are:

1. static analysis (linear and nonlinear) and

2. dynamic normal modes.

The static analysis will include mechanical load environments (pressures) and/or thermal load environments (in-depth thermal gradients). The load/temperature values defined for these analyses are provided by the TAP II aerothermal simulation module solution results. Automated procedures to interpolate the TAP II results to the structural FEM model are provided by the MSC/PATRAN FEM field interpolation function. The transparency FEM may include "contact only" nonlinear elements in the regions of attachment to simulate joint details. The nonlinear iterative procedures to establish the discontinuous contact elements' unique combination of contact and gapping is provided as one of the solution procedures. The transparency material properties used in these static analysis simulations can be temperature dependent and/or nonlinear in their defined constitutive relationships. The solver must provide the static solution procedures to accommodate temperature dependent element material property evaluations.

The dynamic environments to be simulated by for the transparency design assessments include normal modes evaluations and possibly subsequent frequency response and random vibration solutions. The solver must provide enhanced dynamics analysis capability sufficient to simulate any foreseen transparency dynamic load environment or modal content survey. Results must be provided in either the time or frequency domain. 


\subsection{Bird Impact Response Simulation}

A primary requirement of the ADP is to simulate soft body impact representative of the birdstrike of aircraft transparencies. The analytical simulation of transparency non-linear transient dynamic response to birdstrike represents a distinctive class of impact structural behavior. The University of Dayton Research Institute (UDRI) has developed and is continuing to enhance an explicit non-linear dynamic response FEA code, X3D (References 12, 13 and 14). X3D utilizes an explicit solution approach similar to other well-known FEA codes; e.g., DYNA3D and ABAQUS Explicit. These solution methods and codes have been widely used for the numerical simulation of a variety of shock and wave propagation problems. Impact problems in general can be dominated by complicated contact surface conditions which can require very small numerical integration time steps which in turn diminish the solution advantages of an implicit solution approach.

X3D provides functionality to idealize both the bird (impactor) and the transparency (target) and to allow them to come into transient contact with the physics of momentum transfer defined by the non-linear explicit solution algorithm solution results. That is, no analysis assumptions regarding contact pressure time histories or spatial distributions are required.

X3D has been evaluated through executions and correlations with benchmark test cases; i.e., impacting Taylor cylinder, exploding cylindrical shell, and F-16 centerline transparency impact. Although documented validation problems are limited, those completed to-date show promising correlations and demonstrate the improved simulations possible with the use of X3D code.

The X3D impact dynamics code provides a significant numerical tool kit to simulate the complex soft body impact environment of transparency birdstrike:

1. FEA Elements

- Both 3D solid (HEX and TET) and 2D layered shell elements are provided. The 2D layered shell element accommodates soft interlayers (plane selections do not remain plane over the overall layered shell thickness)

2. Material Models (2D)

- Elastic-Plastic, Rate Sensitive, Isotropic

- Linear Elastic, Orthotropic, Brittle Failure

- Viscoelastic with failure

3. Material Models (3D)

- Elastic-Plastic, Rate Sensitive, Isotropic

- Same as above with Discontinuous P-V

- Newtonian Viscous Fluid

4. Automated Contact Surface Evaluation Procedures

- Slave and Master node sets that are nonlinearly evaluated for contact

5. Simple "Rigid Wall" designation procedures

6. Linked element lists for failure assessments

7. Element and integration stabilization features

An example X3D result is shown in Figure 7. The deformed transparency and bird finite element models are displayed, for the specified time point. A fringe plot of Von Mises stress is displayed on the model. 


\subsection{Optical Performance Simulation}

Optical performance is a critical aspect of aircraft transparency design. To support such design the Air Force funded the development of the OPTRAN solver which was specified for inclusion into the ADP. OPTRAN is a raytrace code which evaluates the optical quality of aircraft transparencies subjected to operational load conditions. The code was developed by the University of Dayton Research Institute, References 15 and 16.

The raytrace optical code can be interfaced to finite element thermal and stress analysis codes to permit the effects of operational loads to be modeled. Thermal, displacement, and stress field definition data computed by the finite element codes are input to the optics module. This information is required to compute the orthotropic indices of refraction throughout the material volume of the aircraft transparency. This computation is performed at each step along the propagation path of each ray.

The optics code tracks rays of various wavelengths through the transparency. The deformed geometry generated by the stress analysis is used to determine angles of reflection and refraction at transparency layer boundaries. Birefringent indices of refraction are computed as a function of material, temperature and stress state at the refracting surfaces and within the transparency material.

Key results include angular deviation, transmittance, and polarization effects over specified regions of the transparency. Displacement vectors and deformed grids can also be generated. An example OPTRAN result is shown in Figure 8. The result shows a ray trace for a path originating at the pilots eye, through the head-up display (HUD) and through the transparency.

The integration of OPTRAN in the ADP (Version 2.1) is limited to the case of unloaded/undeformed transparency. The primary intent of the initial implementation was to support basic optical design.

\subsection{Injection Molding Process Simulation}

The C-MOLD product is a family of computer codes designed to support the design of tooling and specification of process parameters for fabrication by injection molding, Reference 17. The modular product supports both the processing of thermoplastic and reactive materials. CMOLD is a commercial-off-the-shelf (COTS) product developed and marketed by AC Technology, Ithaca, NY. AC Technology also provides materials characterization services and maintains a materials database relevant to the injection molding of plastics.

The frameless transparency development is concerned with the use of thermoplastic materials. Therefore, there are three primary processes requiring simulation. These are:

1. the process of the initial filling of the mold (C-FLOW),

2. the post-filling process where shrinkage occurs (C-PACK), and

3. the transient cooling of the part prior to mold separation (C-COOL).

The product modules which address these processes are defined in parentheses above.

The C-FLOW analysis models the mold filling process as a generalized Hele-Shaw (very slow motion) flow (Reference 17). The flow conditions are for an incompressible viscous polymeric melt under non-isothermal conditions and symmetric thermal boundary conditions. The numerical solution is based on a hybrid finite-element/finite-difference method to solve the pressure and temperature fields, and a control-volume method to track moving melt fronts. Details of the analysis methodology are presented in References 18 and 19. 
The C-PACK analysis module extends the above analyses to include the effects of asymmetric thermal boundary conditions. A set of unified governing equations for the flowfield is used throughout the filling and post-filling stages. The analyses can model a three-dimensional, thin cavity with a melt-delivery system that may contain cold or hot, circular or non-circular runners. The influence of shrinkage is also included. Details relevant to the C-PACK analysis are presented in References 20, 21 and 22.

$\mathrm{C}-\mathrm{COOL}$ is a three-dimensional mold cooling simulation to assist in designing the cooling channel system for plastics injection molding processes. The capability exists to model a homogeneous, three-dimensional mold with a thin cavity and with a cooling system that contains circular or non-circular channels, baffles and bubblers. A channel network analysis within the program within $\mathrm{C}$-COOL predicts flow rates in different cooling lines.

The C-COOL module uses a strategy which minimizes input data requirements, user time and computer memory requirements. Heat transfer within the polymer melt is treated as transient, local, one-dimensional heat conduction with static solidification. Heat transfer within the mold is treated as transient, three-dimensional conduction. Heat exchange between the channel surfaces and the cooling fluid is treated as steady and is accounted for using correlations for the convective heat transfer coefficient. To solve the relevant governing equations simultaneously, C-COOL uses a hybrid scheme consisting of a modified, three-dimensional boundary element for the mold region and a finite difference method with a variable mesh for the melt region. These two analyses are coupled iteratively to match the temperature and heat flux at the mold/melt interface. A specialized algorithm has been developed which reduces the computational memory requirements by a factor of 100, compared to the requirements for a traditional approach.

The ADP interface with C-MOLD was primarily limited to the roles of preference access and file management. IGES input files and C-MOLD results files were managed to maintain analysis pedigree. The full functionality of C-MOLD including its GUI, modeling methodology, material data management, and post-processing capability was retained. The C-MOLD GUI is reasonably compatible with that of MSC/PATRAN providing a smooth inter product look and feel.

An example C-MOLD result is shown in Figure 9. Shown is a result from the C-FLOW module, which shows the melt front as a function of time during the mold filling process. The example modeled the Configuration Frameless Transparency, using a representative polycarbonate resin.

\subsection{CONCLUDING REMARKS}

ADP (Version 2.1) has been designed specifically to support the Air Force Frameless Transparency Program. The completion of the development has provided an important suite of tools to aid in the design and performance evaluation of injection molded transparency systems. The validation of these tools will be a critical aspect of the final development process.

ADP provides a fully integrated methodology relevant to the general problem of aircraft transparency design. A key aspect of the ADP is the seamless integration of the analysis modules with MSC/PATRAN, an advanced commercially supported CAE integration tool. This integration provides a single form driven user interface which serves to manage the analysis process and the analysis module input and output (results) data. The user remains in an intuitive environment and is freed of the complexities and/or peculiarities of the computer operating system throughout the entire design process. The support provided by the employment of a commercial CAE integration tool and the selection of state-of-the-art application modules will provide Air Force and its contractors with a cost effective tool with a significant life potential. 


\subsection{REFERENCES}

1. Turner, D.L., "Interim Report Draft III: Development Plan for Frameless Transparency Analytical Design Package," Contract No. F33657-84-C-0247, CDRL No. 34002, Report No. FZM-719-001, General Dynamics Corp., Ft. Worth, TX; 28 June 1990.

2. Hunten, K.A., "Analytical Design Package Development Report," Contract No. F33657-84-C-0147, CDRL No. 34002, Report No. FZM-719-009, General Dynamics Corp., Ft. Worth, TX; 21 November 1991.

3. Hunten, K.A., Analytical Design Package Version 1.2c Operation Manual, Contract No. F33657-84-C-0247, CDRL No. 34002, Report No. FZM-719-010, General Dynamics Corp., Ft. Worth, TX; 22 November 1991.

4. P3/PATRAN User Manual, Vols. 1, 2, 3, 4, Publication No. 903000, PDA Engineering, Costa Mesa, CA; September 1992.

5. M/VISION, Materials Visualization, Selection and Data Integration, User Manual, Publication No. 2190011, PDA Engineering, Costa Mesa, CA; September 1990.

6. Mack, T.E., Kipp, T.E., Whitney, T.J., and Gran, M., "The Use of Computerized Materials Data in ADP," Conference on Aerospace Transparent Materials and Enclosures. (Sixteenth), U.S. Air Force Wright Laboratories; 9-13 August 1993.

7. Varner, M.O., et al, "Specific Thermal Analyzer Program for High Temperature Resistant Transparencies for High-Speed Aircraft (STAPAT)," Report No. AFWAL0TR-84-3086, Volumes I, II and III, AD B089 497, AD B1090 894L, and AD B090 896L, Wright Research and Development Center, Wright-Patterson AFB, OH; October 1984.

8. Bowman, B.L., "Hypersonic Thermal Analysis for Aircraft Transparencies, Vol. I: STAPAT II Description," Report No. WRDC-TR-90-3053, Wright Research and Development Center, Wright-Patterson AFB, OH; September 1990.

9. Bowman, B.L., "Hypersonic Thermal Analysis for Aircraft Transparencies, Vol. II: STAPAT II User's Manual," Report No. WRDC-TR-90-3053, Wright Research and Development Center, Wright-Patterson AFB, OH; September 1990.

10. Lahey, R.S., et. al., MSC/NASTRAN Reference Manual, Version 68 Volumes I, II, III, The MacNeal-Schwendler Corporation, Los Angeles, CA, 1994.

11. P3/FEA Application Module User Manual, Publication No. 903006, PDA Engineering, Costa Mesa, CA; September 1992.

12. Brockman, R.A. and Held, T.W., "Explicit Finite Element Method for Transparency Impact Analysis," Report No. WL-TR-91-3006, Wright Laboratory, WrightPatterson AFB, OH; June 1991.

13. Brockman, R.A. and Held, T.W., "X3D--3D Explicit Finite Element Analysis-Tutorial," University of Dayton Research Institute, Dayton, OH; July 1991.

14. Brockman, R.A. and Held, T.W., "X3D Users' Manual, Updated for X3D Version 3.04," Report No. UDR-TR-92-59, University of Dayton Research Institute, Dayton, OH; April 1992. 
15. Fielman, J.W. and Loomis, J.S., "Optical Analysis of Aircraft Transparencies (OPTRAN), Volume II: Theoretical Manual," Report No. WRDC-TR-90-3058, Volume I, Wright-Research and Development Center, Wright-Patterson AFB, OH; October 1990.

16. Fielman, J.W. and Loomis, J.S., "Optical Analysis of Aircraft Transparencies (OPTRAN), Volume I: OPTRAN User's Manual," Report No. WRDC-TR-90-3058, Volume I, Wright-Research and Development Center, Wright-Patterson AFB, OH; June 1990.

17. CMOLD Reference Manual, AC Technology, Ithaca, NY; 1992.

18. Hieber, C.A. and Shen, S.F., "A Finite Element/Finite-Difference Simulation of the Injection Molding Filling Process, Journal of Non-Newtonian Fluid Mechanics, Vol. 7; 1980 .

19. Wang, V.W., Hieber, C.A., and Wang, K.K., "Dynamics Simulation and Graphics for the Injection Molding of Three-Dimensional Thin Parts," Journal of Polymer Engineering, Vol. 7, No. 1; 1986.

20. Chiang, H.H., et al, "Integrated Simulation of Fluid Flow and Heat Transfer in Injection Molding for the Prediction of Shrinkage and Warpage," Vol. ASME-HTD175/MD-25, pp. 133-146; 1991.

21. Chiang, H.H., Hieber, C.A., and Wang, K.K., "A Unified Simulation of the Filling and Postfilling Stages in Injection Molding. Part 1: Formulation and Part 2: Experimental Verification," Polym. Eng. Sci., Vol. 31, pp 116-139; 1991.

22. Hieber, C.A., Chapter 1 in Injection and Compression Molding Fundamentals, A.I. Isayev, Ed., Marcel Dekker, New York; 1987. 
Table 1. ADP Development Phases

\begin{tabular}{|l|c|c|}
\hline \multicolumn{1}{|c|}{ MODULE FUNCTION } & ADP (Version 2.1) & ADP/COTS \\
\hline \hline $\begin{array}{l}\text { Design Process Manager, } \\
\text { Graphical User Interface, } \\
\text { and Project Manager }\end{array}$ & MSC/PATRAN & MSC/PATRAN \\
\hline Modeling/Post-Processing & & MSC/PATRAN \\
\hline CAD Interface & MSC/PATRAN & MSC/PATRAN \\
& MSC/PATRAN & MSC/PATRAN CFD \\
\hline Aeroheating Environment & Sable 2) & STAHET \\
\hline Thermal Response & TAP & MSC/NASTRAN \\
\hline Structural Analysis & MSC/NASTRAN & MSC/PATRAN FEA \\
\hline Birdstrike Analysis & MSC/PATRAN FEA & CBAUS EXPLICIT \\
\hline Optical Raytrace & OPTRAN & CODE V \\
\hline Injection Molding & C-MOLD 4.0 & MSC/MVISION \\
\hline Materials Properties and & MSC/MVISION & MSC/PATRAN \\
Process Data Management Sys. & MSC/PATRAN & MATL. SELECTOR \\
\hline Results Animation & MATL. SELECTOR & MSC/PATRAN ANIMATION \\
\hline
\end{tabular}

Table 2. Application Modules Commercially Integrated With MSC/PATRAN

\begin{tabular}{|c|l|}
\hline MODULE FUNCTION & MODULE TRADE NAME \\
\hline \hline \multirow{3}{*}{ CAD INTERFACE } & UNIGRAPHICS \\
& CADDS 5 \\
& CATIA \\
& Pro/ENGINEER \\
& EUCLID-IS \\
& IGES \\
& PDES/STEP \\
\hline THERMAL RESPONSE & MSC/PATRAN THERMAL \\
& SINDA \\
& MSC/PATRAN ADVANCED FEA \\
& ABAQUS \\
\hline STRUCTURAL ANALYSIS & ABAQUS \\
& ANSYS \\
& MARC \\
& MSC/NASTRAN \\
& MSC/PATRAN FEA \\
& MSC/PATRAN ADVANCED FEA \\
\hline
\end{tabular}


Table 3. General Capabilities of STAHET Solver

\begin{tabular}{|l|l|}
\hline \multicolumn{1}{|c|}{ STAHET I } & \multicolumn{1}{c|}{ STAHET II ENHANCEMENTS } \\
\hline \hline - $\begin{array}{l}\text { Two Streamline Tracing Methods } \\
\text { - Modified Newtonian Pressure } \\
\text { Calculation }\end{array}$ & $\begin{array}{l}\text { Simplified Streamline Tracing Techniques } \\
\text { Selected as Default }\end{array}$ \\
- Laminar and Turbulent Heating Correlations & $\begin{array}{l}\text { User Input of Streamline Starting Angles } \\
\text { Added }\end{array}$ \\
- Boundary Layer Transition Options & $\begin{array}{l}\text { Four Shadow Region Pressure Prediction } \\
\text { Techniques Added }\end{array}$ \\
- Wall Temperature Effect Modeling & Mission Profile (Mach and Altitude) \\
Input for 3-D Geometries & Wall Temperature Effect Modeling \\
2-D Wind Tunnel Modeling & 3-D Wind Tunnel Model Capability Added \\
Standard Day, Hot Day, Cold Day & Extension to Hypersonic Flow \\
Atmospheres & \\
\hline
\end{tabular}

Table 4. General Capabilities of TAP Solver

\begin{tabular}{|l|l|}
\hline \multicolumn{1}{|c|}{ TAP I } & \multicolumn{1}{c|}{ TAP II ENHANCEMENTS } \\
\hline \hline - 3-D Finite Element Solution & - Defog Modeling Improved \\
- Material Property Data Base & - Cabin Cooling Air Velocity Default Added \\
- Aeroheating Imposed on External Surface & - Fluid Gap Modeling Improved \\
- Thermal Boundary Conditions & - External Anti-Icing Improved \\
- Generalized Convection & - Line Source Capability for Convection Added \\
- Anti-Ice (Hot Air Blast) & - Earth Radiation Sink Temperature Added \\
- Electrical Anti-Ice & - Expanded Material Property Data Base \\
- Radiation-to-Sky & - Viscosity and Molecular Weight Added to \\
- Element-to-Element Radiation & Material Property Data Base \\
Standard Day, Hot Day, Cold Day & - Gap Fluids Added to Material Property \\
Atmospheres & Data Base \\
Mission Profile (Mach, Altitude and Time) & - Extension to Hypersonic Flow \\
\hline Input &
\end{tabular}




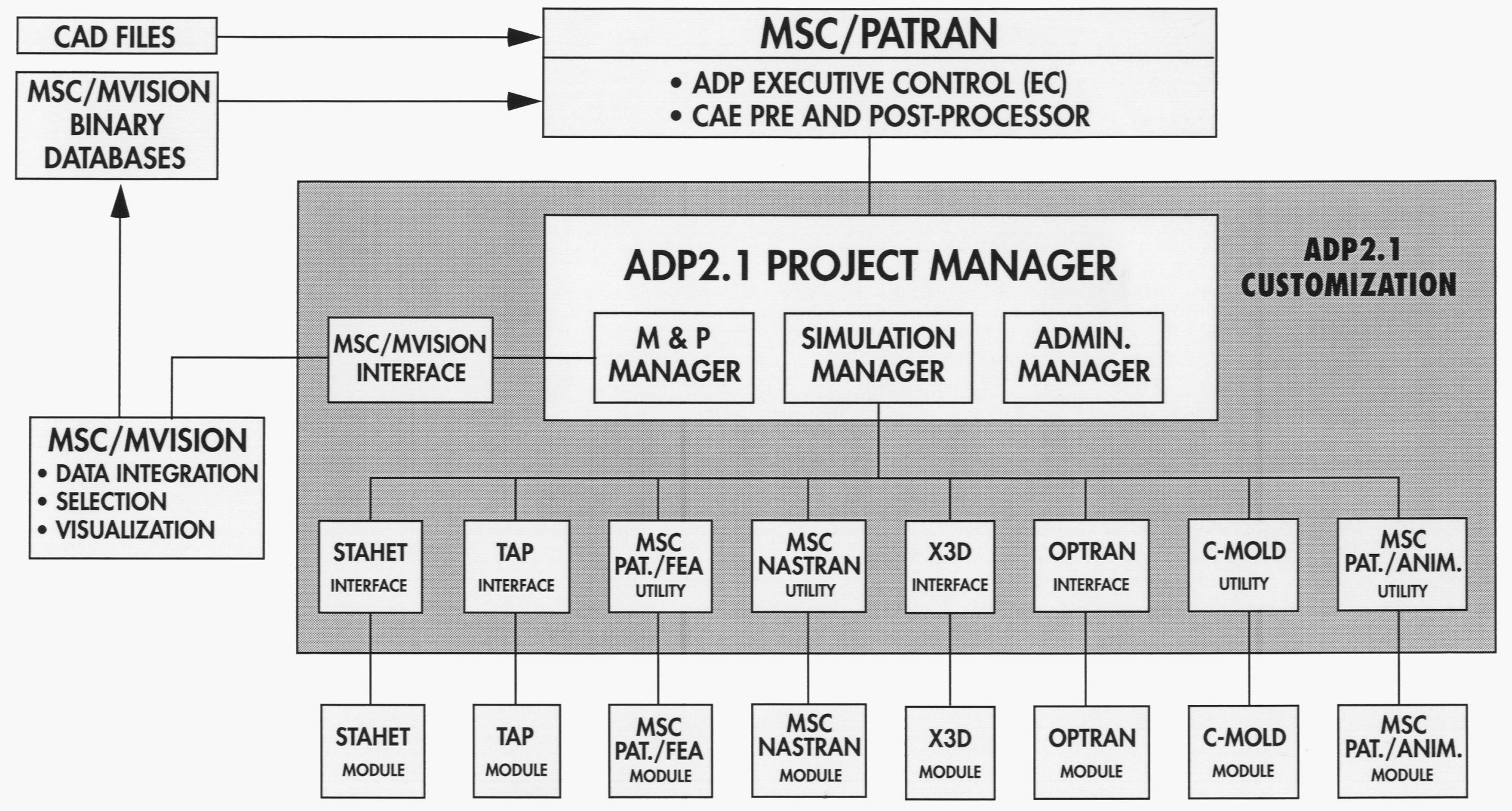

Figure 1. ADP (Version 2.1) Architecture 


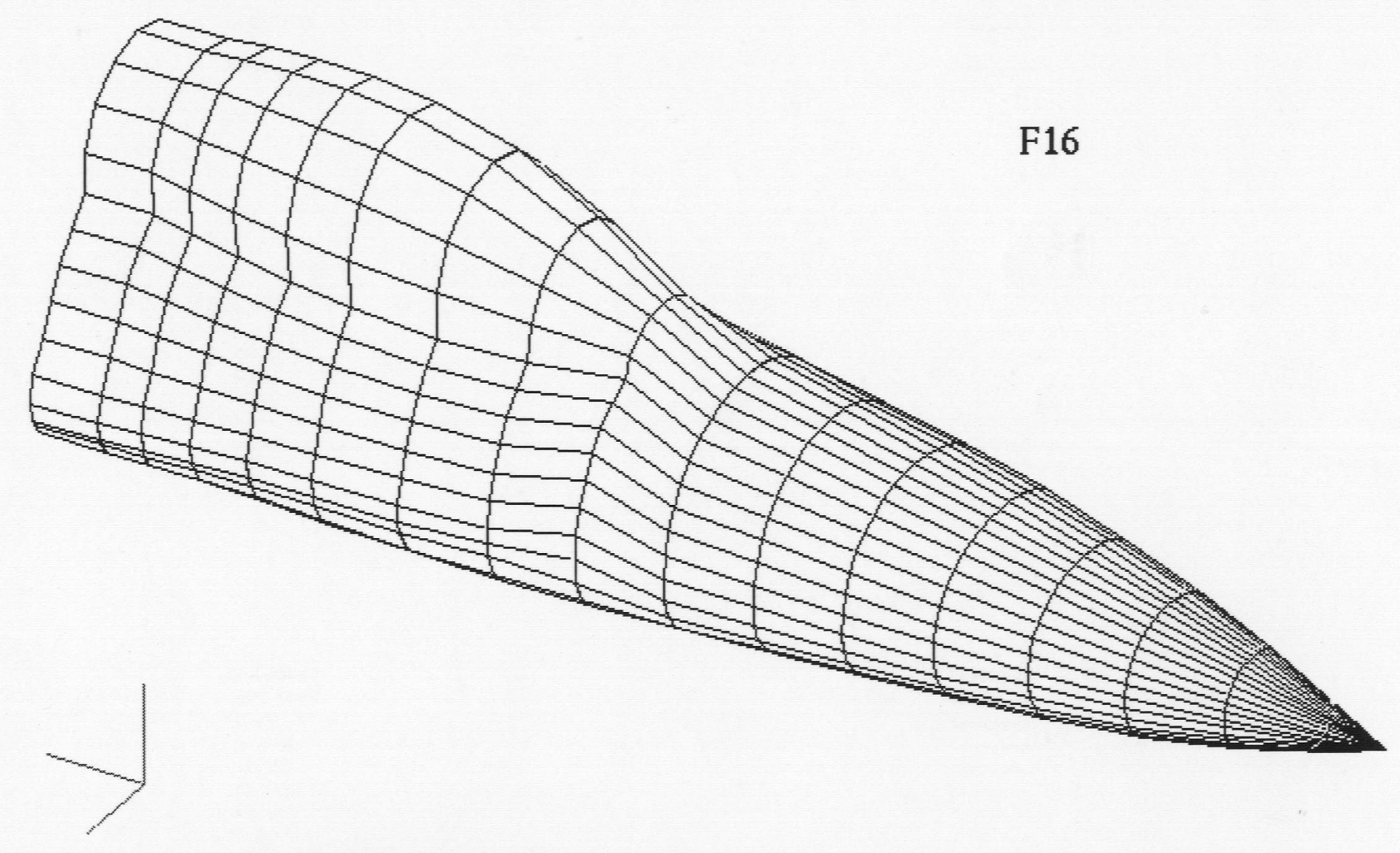

Figure 2 F-16 Aircraft Forebody Model, S-10 Library 


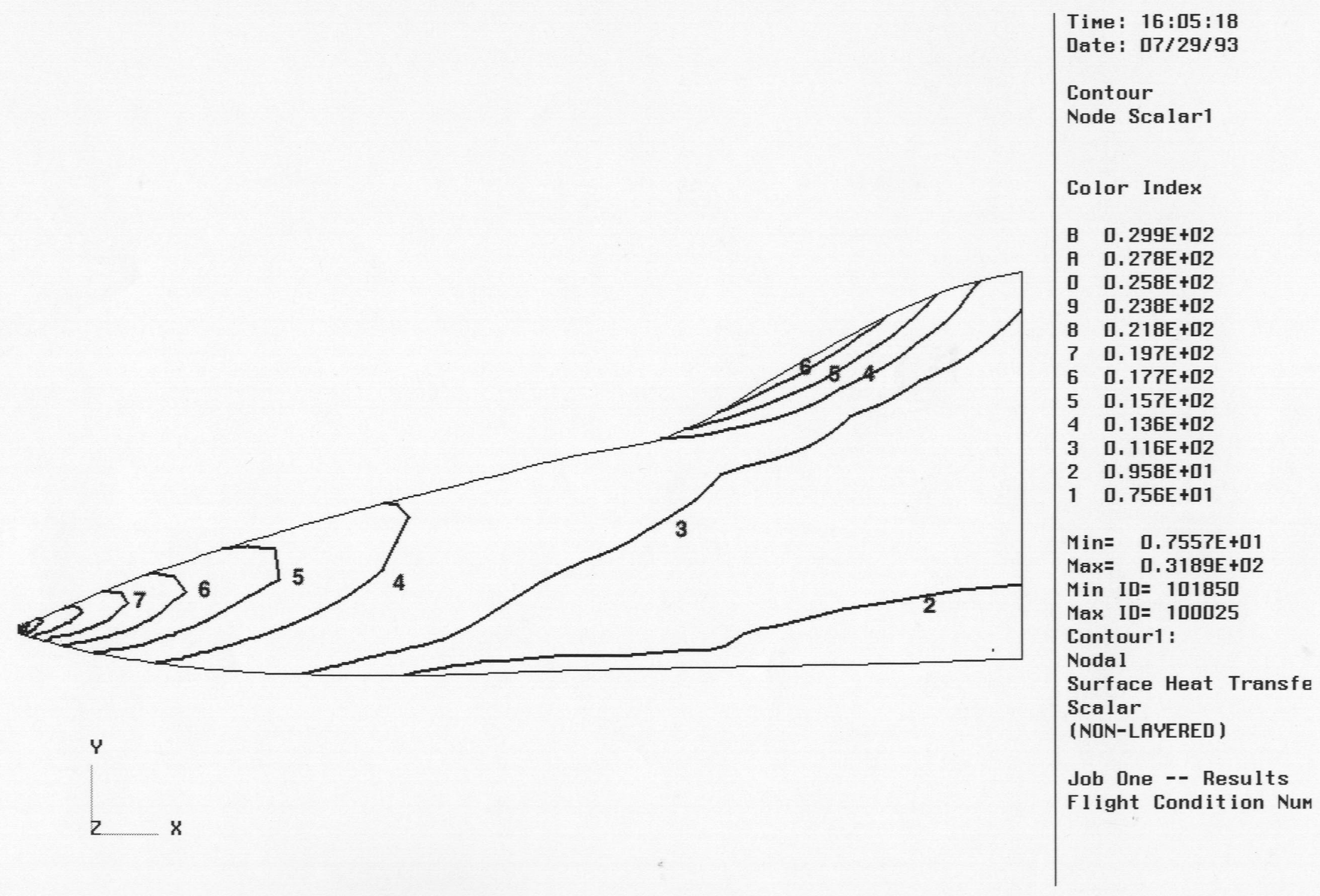

Figure 3 STAHET II Result, Aerodynamic Heating Rate (Btu/ft $\left.{ }^{2} \mathrm{sec}\right)$ 


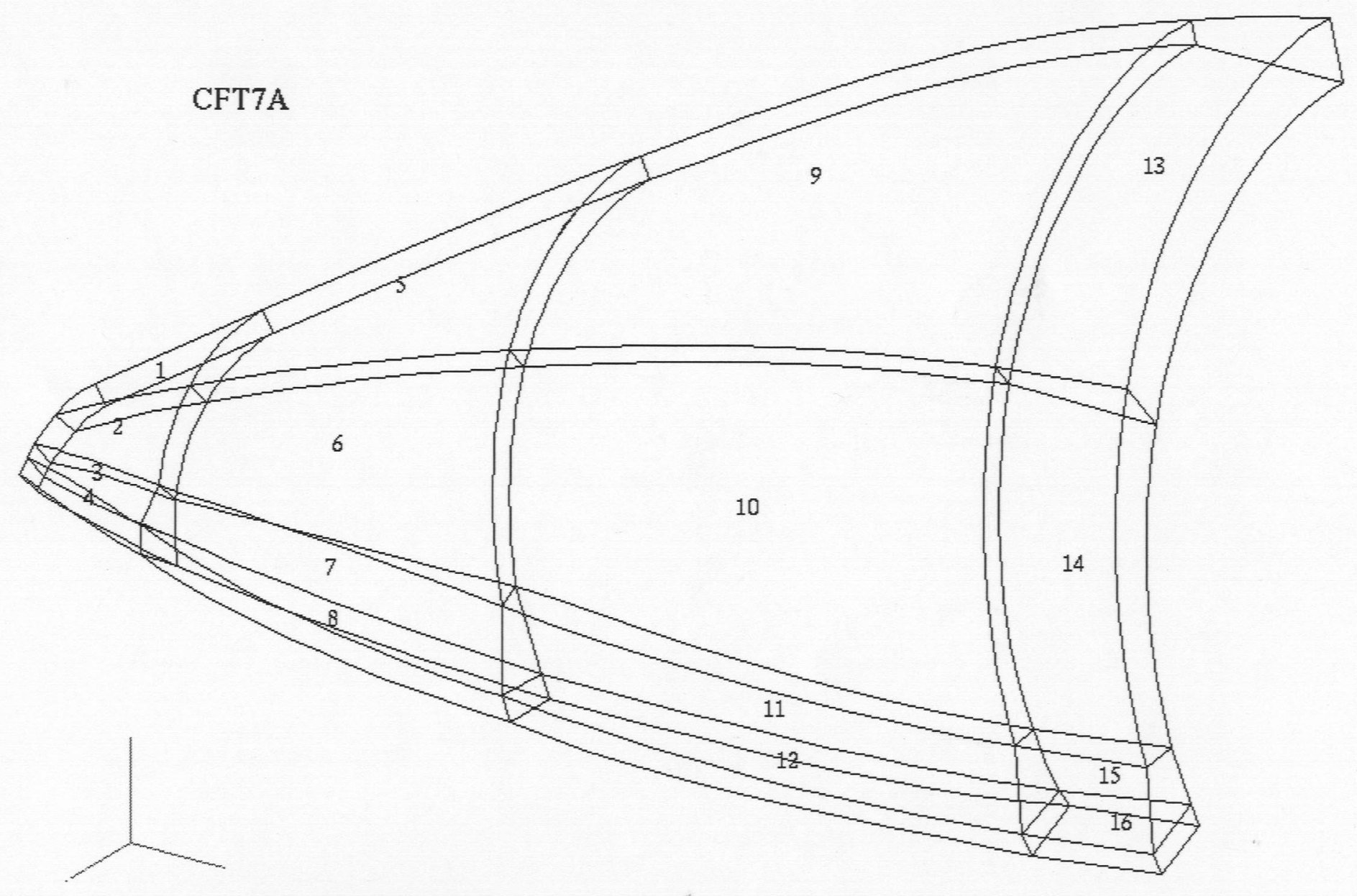

Figure 4 CFT7A Transparency Geometry Model 


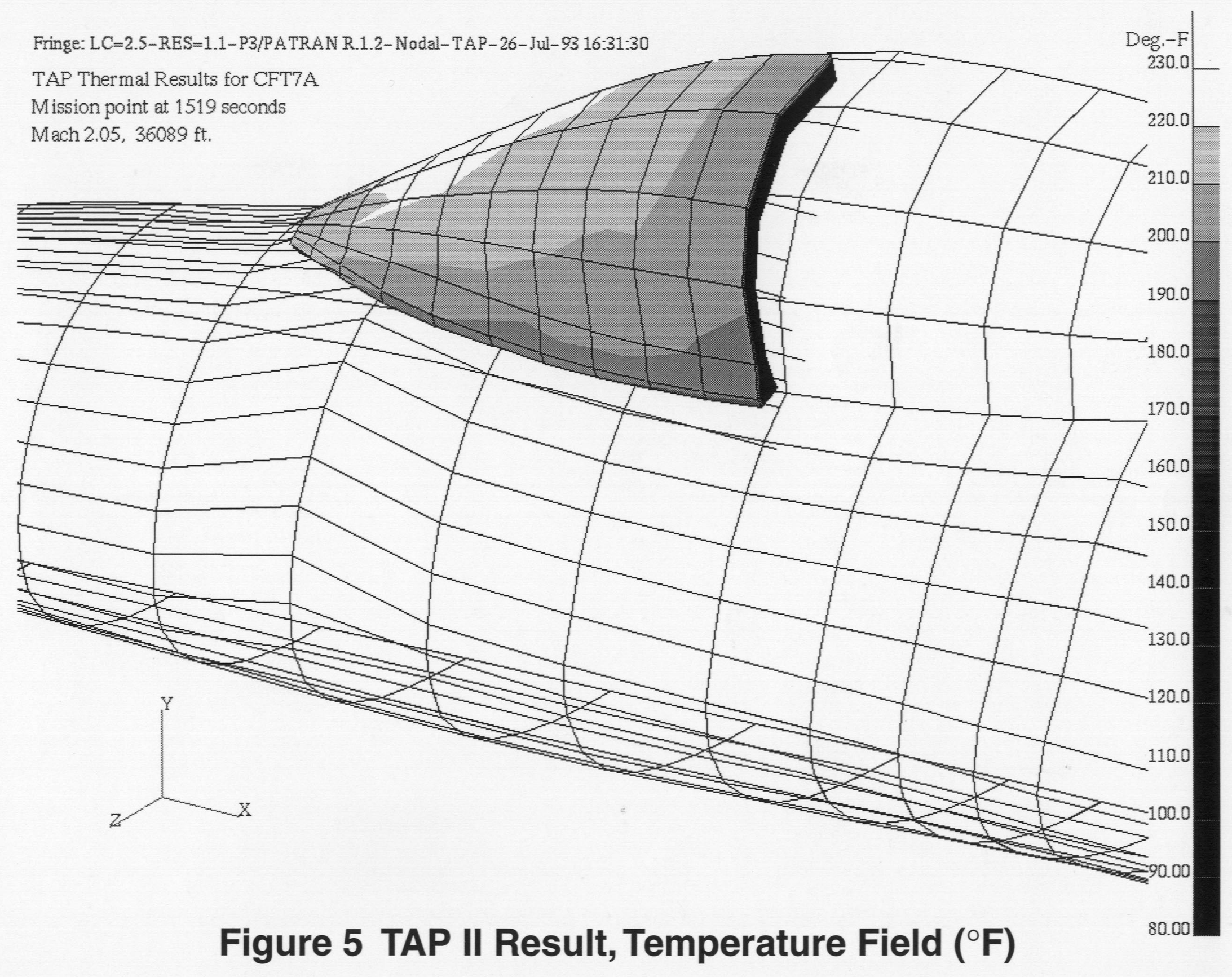



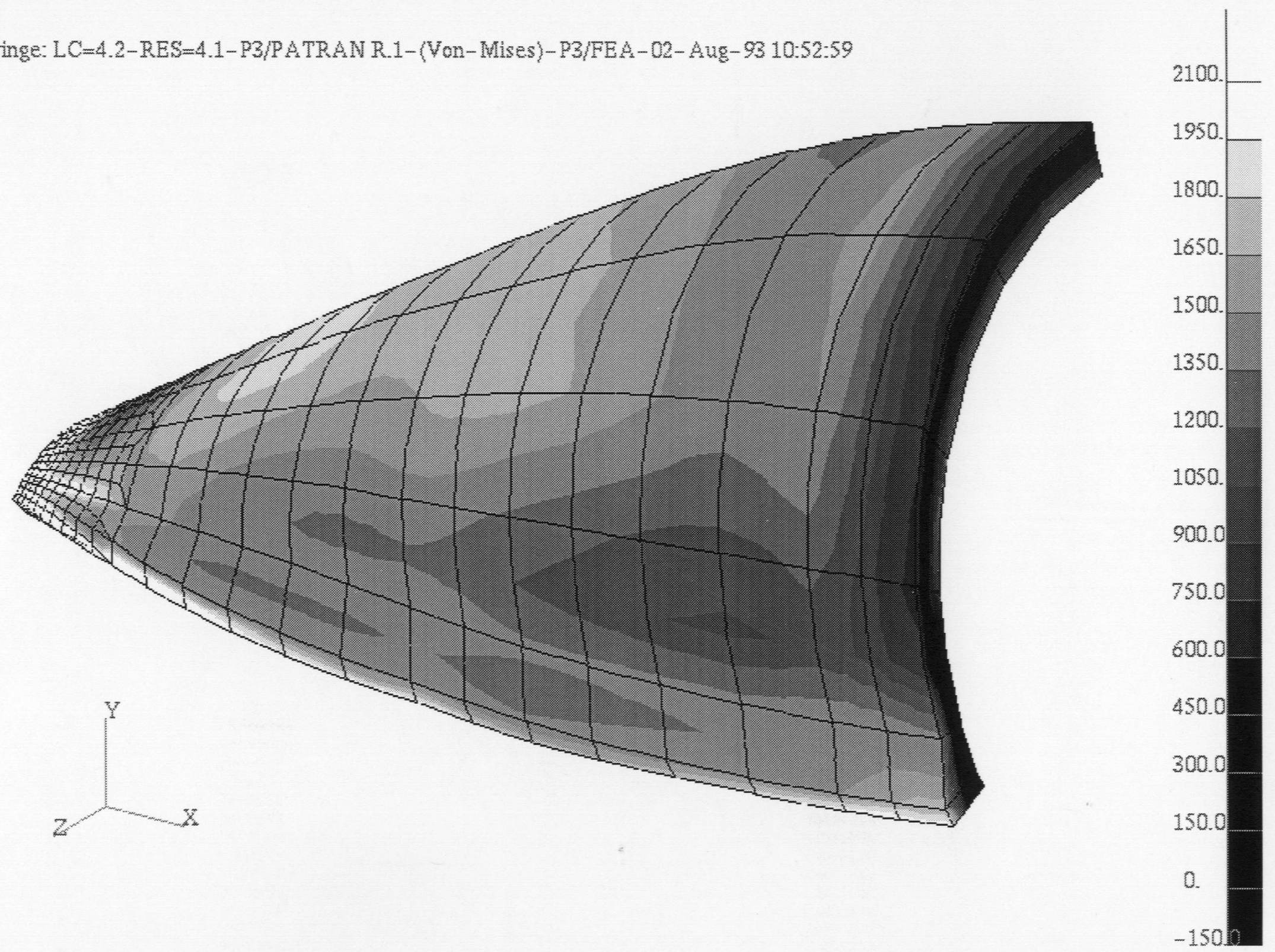

Figure 6 P3/FEA Result, Von Mises Stress, Static Case (psi) 


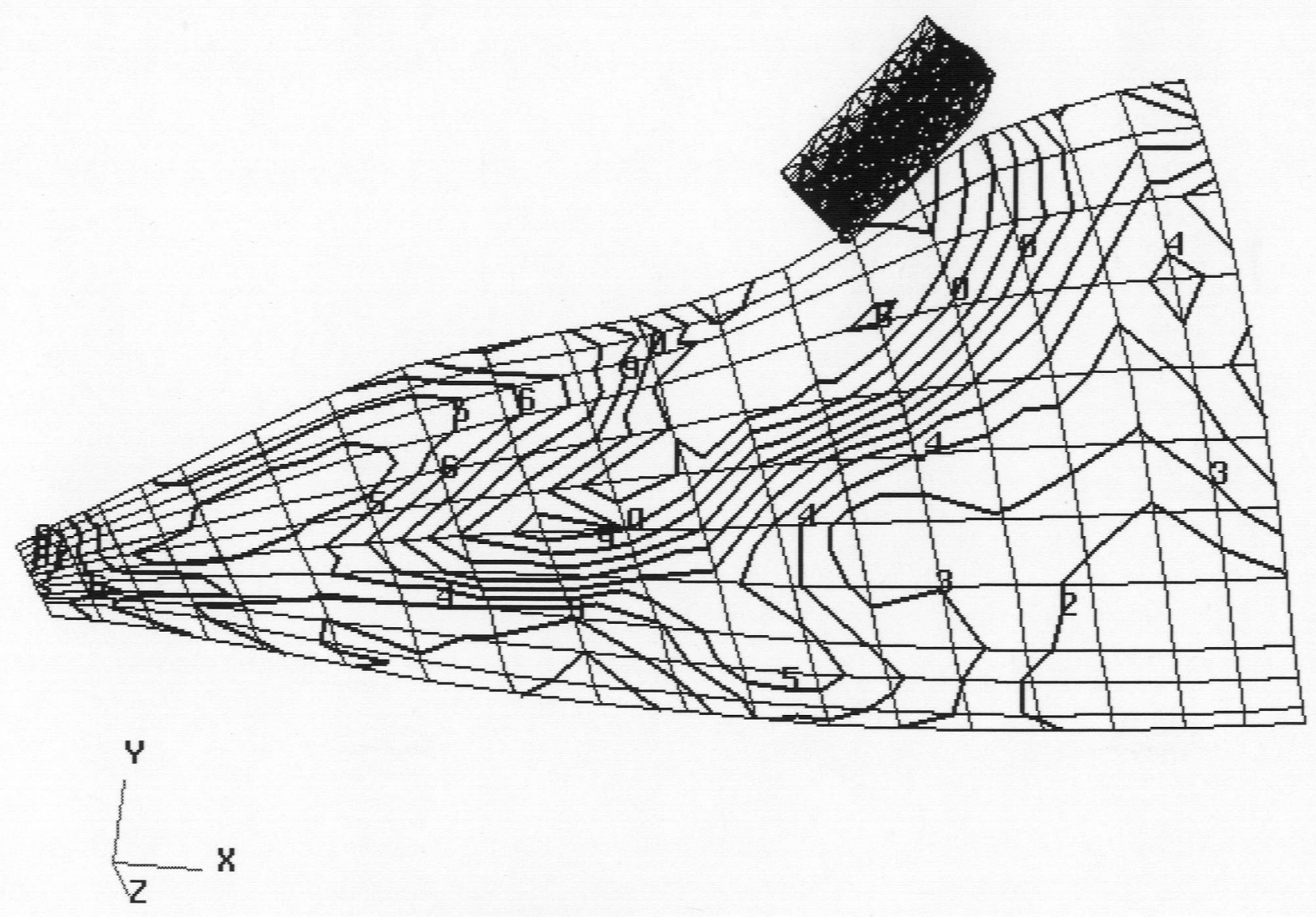

D. $960 \mathrm{E}+04$

D. $883 \mathrm{E}+\mathrm{D} 4$

0. $805 \mathrm{E}+04$

D. $728 \mathrm{E}+\mathrm{D} 4$

D. $651 \mathrm{E}+\mathrm{D} 4$

$0.573 E+04$

0. $496 \mathrm{E}+04$

D. $418 \mathrm{E}+\mathrm{D} 4$

0. $341 \mathrm{E}+\mathrm{D} 4$

D. $263 \mathrm{E}+\mathrm{D} 4$

D. $186 \mathrm{E}+04$

D. 109E+04

Min ID= 42

Max ID= 49

Contour1:

Centroidal

Uon Mises Stress

Scalar

D- (NON-LAYERED )

First Run

Time D.003

Max, Deformat ion =

2. $046820 \mathrm{E}+01$

gNode 292

Figure 7 X3D Result, Time Resolved Von Mises Stress (psi) 


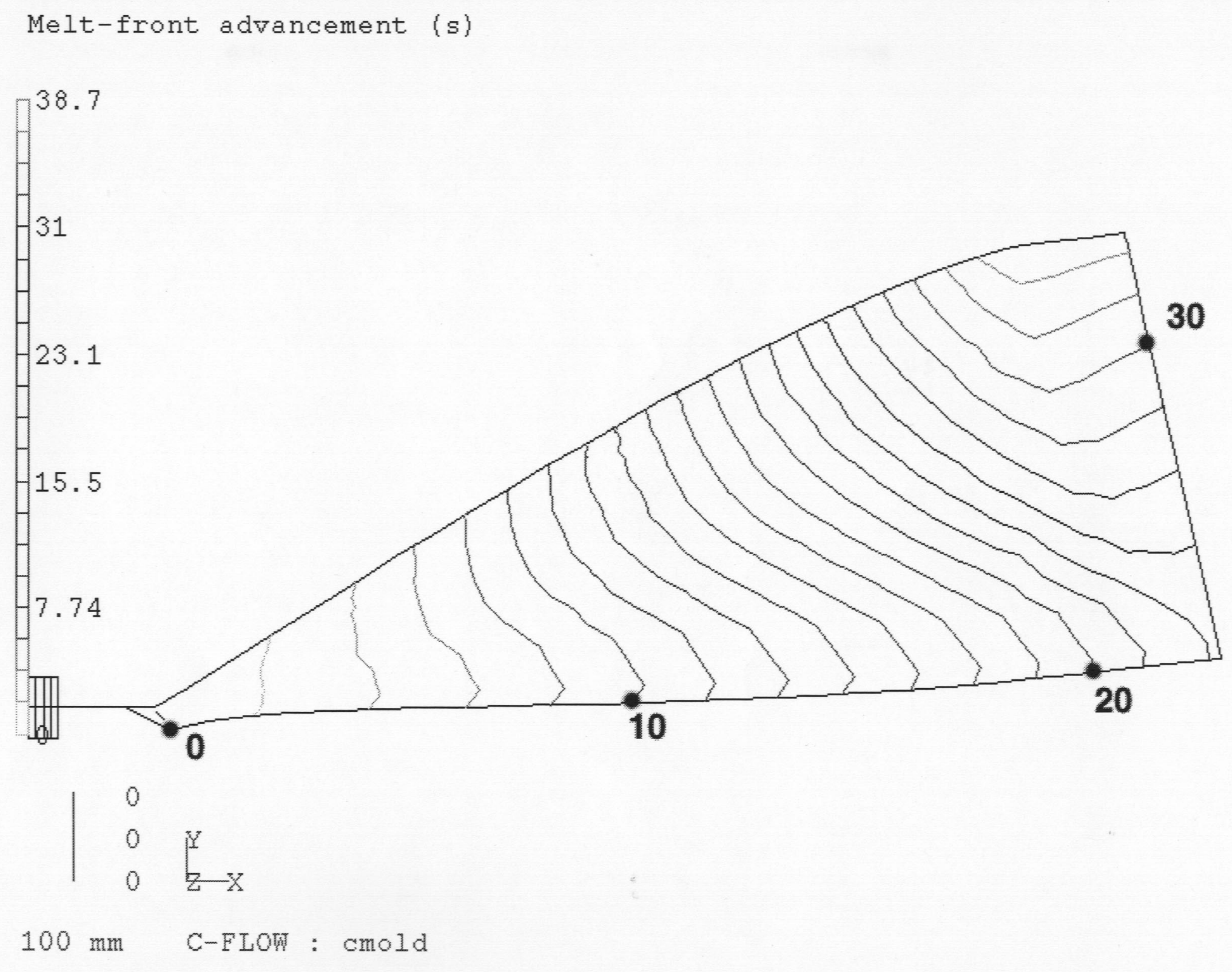

Figure 8 C-MOLD Result, Melt Front Advancement (sec) 


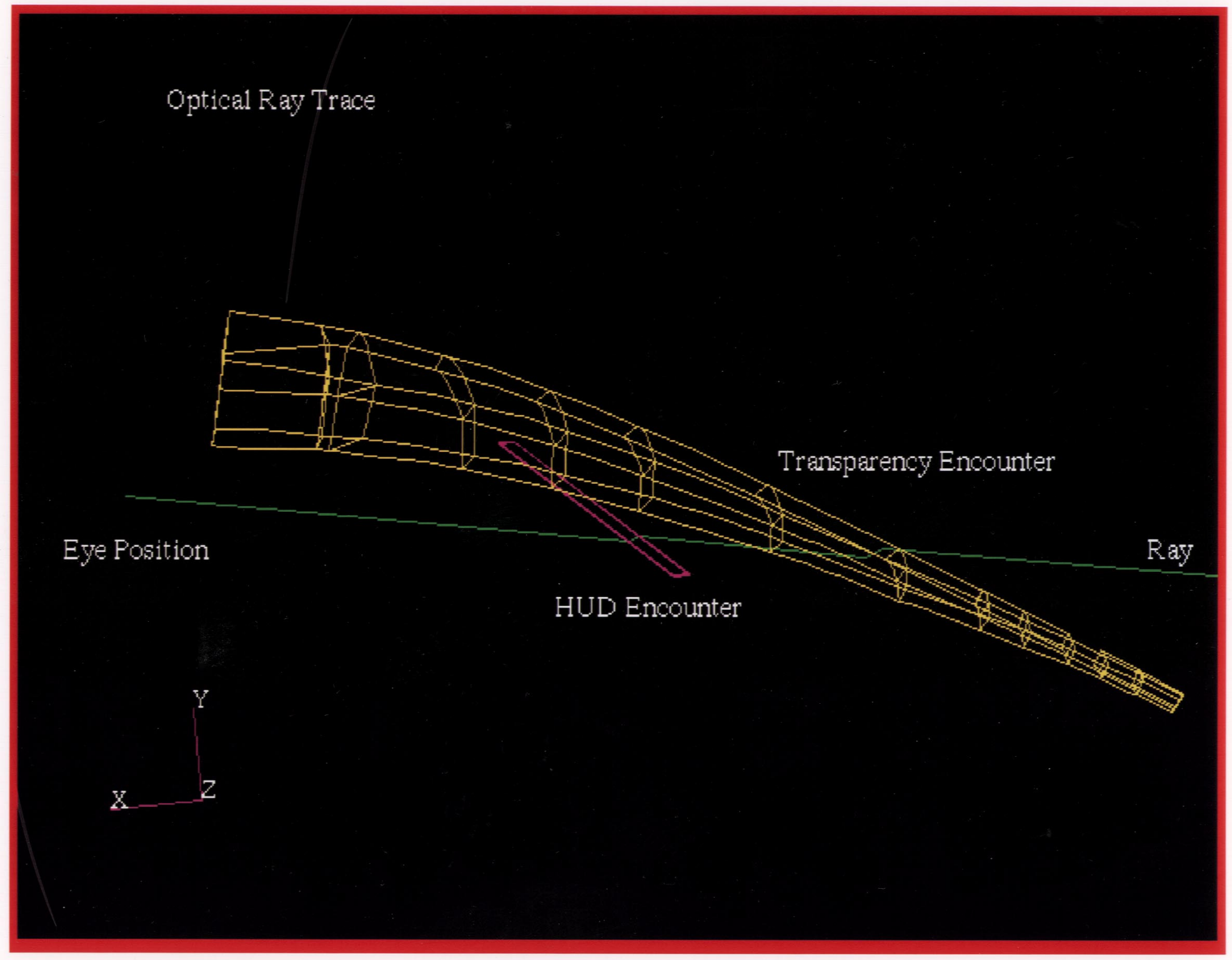

Figure 2.18. OPTRAN Result, Ray Trace Analysis. 